This document is the accepted manuscript version of the following article: Hafer-Hahmann, N., \& Vorburger, C. (2020). Parasitoids as drivers of symbiont diversity in an insect host. Ecology Letters.

https://doi .org/10.1111/e1e.13526

\title{
1 Parasitoids as Drivers of Symbiont 2 Diversity in an Insect Host
}

\section{Authors: Nina Hafer-Hahmann ${ }^{1}$ \& Christoph Vorburger ${ }^{1,2}$}

5 1: EAWAG, Swiss Federal Institute of Aquatic Science and Technology, Überlandstrasse 133, 8600

6 Dübendorf, Switzerland

7 2: Institute of Integrative Biology, ETH Zürich, Universitätsstrasse 16, 8092 Zürich, Switzerland

E-mail: NH: nina.hafer@eawag.ch; CV: christoph.vorburger@eawag.ch

Running title: Parasitoids as Drivers of Symbiont Diversity

Keywords: Parasitoid wasps, aphids, Aphis fabae, Lysiphlebus fabarum, defensive symbiois, experimental evolution, immune system, specificity, adaptation, maintenance of diversity

Type of article: Letter

Number of words in the abstract: 149

19 Number of words in the main text: 4556

20 Number of references: 80

21 Number of figures: 4

22 Number of tables: 2

23 Number of text boxes: 0

Corresponding author: Nina Hafer-Hahmann, EAWAG, Swiss Federal Institute of Aquatic Science and

Technology \& Institute of Integrative Biology, Überlandstrasse 133, 8600 Dübendorf, Switzerland; Phone:

Statement of authorship:

Both authors designed the study. NH conducted the experiment and analysed the data with input from CV.

$\mathrm{NH}$ wrote the first draft of the manuscript and CV contributed substantially to its revision.

34 Data will be archived on Dryad and a doi will be provided if the article is accepted. 
Immune systems have repeatedly diversified in response to parasite diversity. Many

37 animals have outsourced part of their immune defence to defensive symbionts, which should be affected by similar evolutionary pressures as the host's own immune system. Protective symbionts provide efficient and specific protection and respond to changing selection pressure by parasites. Here, we use the aphid Aphis fabae, its protective symbiont Hamiltonella defensa, and its parasitoid Lysiphlebus fabarum to test whether parasite diversity can maintain diversity in protective symbionts. We exposed aphid populations with the same initial symbiont composition to parasitoid populations that differed in their diversity. As expected, single parasitoid genotypes mostly favoured a single symbiont that was most protective against that particular parasitoid, while multiple symbionts persisted in aphids exposed to more diverse parasitoid populations,

47 which in turn affected aphid population density and rates of parasitism. Parasite diversity may be crucial to maintaining symbiont diversity in nature. 


\section{Introduction}

Genes involved in immune defences are amongst the most diverse in any living organism. This diversity allows their owners to defend themselves against a diverse array of parasites and pathogens (hereafter parasites). In vertebrates, for example, a multitude of different mayor histocompatibility complex (MHC) alleles enables the specific and efficient response to parasites (Bernatchez \& Landry 2003; Wegner et al. 2004; Sommer 2005; Milinski 2006; Piertney \& Oliver 2006). Similarly, the CRISPR/Cas System (Horvath et al. 2010; Bhaya et al. 2011; Garrett et al. 2011; Sorek et al. 2013) or Ig-proteins (Watson et al. 2005) provide a variable component to the immune system of bacteria and insects, respectively. It seems that such diversification of the immune system is a predictable outcome of the host's evolutionary arms race with a diverse array of parasites (Litman et al. 2007; Messier-Solek et al. 2010; Ghosh et al. 2011). The diversity of alleles is so high that a single organism can only express a fraction of these variants. Organisms are selected to express the appropriate variants and diversity depending on the dominant parasites and their diversity (Apanius et al. 1997;

Bernatchez \& Landry 2003; Wegner et al. 2004; Sommer 2005; Milinski 2006; Piertney \& Oliver 2006; Spurgin \& Richardson 2010).

Many animals, especially arthropods, have outsourced some of their defence against parasites to protective symbionts (Brownlie \& Johnson 2009; Flórez et al. 2015). Aphids represent the most prominent example of this. They are frequently infected with a diverse array of heritable bacterial endosymbionts that provide specific and effective protection against natural enemies such as entomopathogenic fungi and parasitoid wasps (reviewed by Oliver et al. 2010, 2014; Vorburger 2014; Guo et al. 2017; Vorburger \& Perlman 2018). Different aphid species and populations vary in their 
symbiont repertoire (reviewed by Oliver et al. 2014; Vorburger 2014; Flórez et al. 2015; McLean et al. 2016; Zytynska \& Weisser 2016; Guo et al. 2017), and the symbionts usually occur at intermediate frequencies. Different symbiont species or different strains of the same symbiont species can co-occur within the same aphid species, population, or even individual (reviewed by Oliver et al. 2014; McLean et al. 2016; Zytynska \& Weisser 2016; Guo et al. 2017), raising the question of how this diversity is maintained. Similar to other forms of immunity, protection by endosymbionts usually comes at a cost to the host. In the absence of parasites, aphids carrying protective symbionts tend to suffer from reduced competitive ability or lower reproduction and longevity (Oliver et al. 2008; Vorburger \& Gouskov 2011; Cayetano et al. 2015; Heyworth \& Ferrari 2015; Parker et al. 2017). Parallels between protective symbionts and other specific immune defences based on diverse nuclear alleles suggest that similar evolutionary forces should be at work (Hafer \& Vorburger 2019). The diversity of parasites may thus drive and maintain the diversity of protective symbionts as well. Indeed, there is good evidence that selection by parasitoids increases frequencies of protective symbionts in aphid populations (Herzog et al. 2007; Oliver et al. 2008; Xie et al. 2015; Käch et al. 2018). This symbiont-conferred resistance seems to be specific. Different strains of the defensive endosymbiont Hamiltonella defensa (Moran et al. 2005) are unequally protective against different parasitoid species (Cayetano \& Vorburger 2015; McLean \& Godfray 2015; Leclair et al. 2016), or even against different genotypes of the same parasitoid species (Rouchet \& Vorburger 2012; Schmid et al. 2012; Cayetano \& Vorburger 2013, 2015; Cayetano et al. 2015; Vorburger \& Rouchet 2016). This implies that different parasitoids may select for different symbionts in their host populations. Here we take a step further to test whether symbiont diversity is indeed maintained by parasitoid diversity, using an 
experimental evolution approach. We exposed experimental aphid populations containing multiple strains of $H$. defensa to more or less diverse populations of parasitoids over multiple generations, and we followed the symbiont frequencies in the aphid populations. The prediction was that symbiont diversity should be maintained under selection by multiple parasitoid genotypes, whereas with only one parasitoid genotype, only the symbiont that provides the best protection against this particular parasitoid should persist. Our findings support these predictions, highlighting the crucial role parasites may play in maintaining the diversity of immune defences, also when provided by symbionts. In the absence of any parasitoids, symbionts decreased and could be expected to be lost eventually due to the costs they impose on their hosts.

\section{Material \& Methods}

\section{Insects}

The black bean aphid, Aphis fabae, is an important pest of broad bean (Vicia fabae) and sugar beet (Beta vulgaris). We used four different lines of a single $A$. fabae clone which originated from a single female collected in St. Margrethen, Switzerland from Chenopodium album in 2006 (clone A06-405). The four lines differed only by their infections with the endosymbiotic bacterium $H$. defensa, a well-known protective symbiont that confers increased resistance against parasitoid wasps in several aphid species (Oliver et al. 2003; Schmid et al. 2012; Asplen et al. 2014). Three lines were created by experimentally introducing one of three genetically distinct $H$. defensa strains by microinjection (strain IDs H15, H76, and H402). The fourth line did not receive any $H$. 
defensa (Cayetano et al. 2015). All aphid lines have been maintained clonally on broad beans at ca. $18-20^{\circ} \mathrm{C}$ and a $16 / 8 \mathrm{~h}$ light/dark regime prior to use in this experiment. As parasitoids we used three asexual isofemale lines of Lysiphlebus fabarum, a specialised and frequent parasitoid of $A$. fabae and other species mostly of the genus Aphis (Tomanović et al. 2018). These lines originated from single females collected near Zürich, Switzerland in 2007 from A. fabae fabae (IL07-64), near Geneva, Switzerland in 2009 from A. fabae cirsiiacanthoides (IL09-348), and from A. fabae fabae near Orbe, Switzerland in 2009 (IL09-369). For simplicity, we refer to these parasitoid wasp lines as W64, W348 and W369 hereafter. We chose lines that were known from previous work to differ in their ability to infect aphids protected by various strains of $H$. defensa. W64 is able to parasitize aphids harbouring H15 and H402 but does poorly on aphids with H76. W369 can parasitize aphids with H15 and, to a lesser extent, H76, but not aphids with H402. W348 can parasitize aphids with H402 and, less successfully, H76, but has never been tested on aphids with H15 (Cayetano et al. 2015; Vorburger \& Rouchet 2016). Since their collection these wasps have been reared on symbiont-free $A$. fabae of a different line than the one used in this study at ca. $22^{\circ} \mathrm{C}$ with a $16 \mathrm{~h}$ photoperiod.

\section{Setup of the selection experiment}

At the start of the experiment, we prepared 30 aphid populations with the same composition in insect rearing cages (24.5 x24.5 x 24.5 cm; BugDorm-4F2222; MegaView Science, Taiwan). The cages contained three pots with ca. 2-weeks old broad bean plants (Vicia faba, 2 plants per pot), and the aphid populations comprised equal proportions of H. defensa-free (H-), H15, H76, and H402 infected aphids. We added 9 adult aphid females of each line to every cage (3 per pot), i.e. 36 adults in total. Aphids could move 
143 freely between plants within their cage. After allowing the aphids to reproduce for four

144 to six days, we applied six different treatments to the aphid populations (five replicate

145 cages per treatment). Treatments consisted either of no wasps (NoWasp), wasps of a

146 single asexual line (W64, W348, or W369), a simultaneous mix with equal proportions

147 of the three wasp lines (WMixSim), or a sequential mix of the three wasp lines, i.e. wasps

148 from a single line in each generation, but alternating lines between generations

149 (WMixSeq; Fig. 1A). In the first generation, the wasp treatments were applied by adding

15012 female wasps of the appropriate line(s) to each cage. This number was increased to

15118 wasps per cage for all subsequent generations. After the addition of wasps, we left

152 the populations undisturbed for 11-12 days (see Table S1 for a detailed overview of the

153 timing in each generation). The wasps have a short lifespan of only two to three days

154 (Satar et al. 2019), and successfully parasitized aphids are killed and become easily

155 recognizable as mummies within 7-9 days after parasitoid oviposition. We then

156 collected 36 healthy adult aphids from each cage to serve as parents of the next aphid

157 generation. To ensure that they were a representative sample of the surviving aphids we

158 took 12 adults from each pot and we took care to take them from different places on the

159 plants to avoid taking offspring of a single mother. If we were unable to obtain 12 aphids

160 from one pot, we collected the remaining aphids equally from the other pots, and we

161 substituted with the oldest nymphs we could find if we were unable to recover 36

162 healthy adults. The collected aphids were used to inoculate plants for the subsequent

163 generation by adding 12 aphids to each new pot in a fresh cage (Fig. 1B). Experimental

164 evolution continued for 10 generations, although we lost one replicate evolution line of

165 treatment WMixSeq, because the aphid population went extinct after 8 generations. 


\section{Data collection}

167 During each transfer, we counted the number of mummies, we roughly estimated the 168 number of aphids by counting aphids in groups of ca. 10 individuals, and we measured 169 the total stem length of all plants in the old cages. Additionally, at the end of the $2^{\text {nd }}, 6^{\text {th }}$, 170 and last $\left(10^{\text {th }}\right)$ generation, we collected 15 unmummified aphids per cage ( 5 aphids from 171 each pot) to estimate aphid population composition. Aphids were stored at $-20^{\circ} \mathrm{C}$ until 172 further analysis.

173 Aphid DNA was extracted using high salt extractions following the protocol in 174 (Sunnucks \& Hales 1994), albeit adapted to a 96 deep well plate format (Gouskov et al. 175 2016). Subsequently, DNA was used for diagnostic PCRs (see Ferrari et al. 2012) to test

176 for the presence of $H$. defensa and the obligate endosymbiont of aphids, Buchnera 177 aphidicola, with symbiont-specific primer pairs amplifying parts of the bacterial 16S 178 rRNA gene. Since B. aphidicola is present in all individuals, this served as a control for 179 the success of the DNA extraction. Samples that were negative for B. aphidicola were 180 discarded. Initially, we conducted separate PCRs for B. aphidicola (forward primer: 181 16SA1; AGAGTTTGATCMTGGCTCAG (Fukatsu \& Nikoh 1998) \& reverse primer: 182 Buch_16S_Afab_R: CTTCTTCGGGTAACGTCAAGAA) and H. defensa (forward primer: 10F: 183 AGTTTGATCATGGCTCAGATTG \& reverse primer: T419R:

184 AAATGGTATTCGCATTTATCG) (Ferrari et al. 2012), but during the course of this work 185 switched to a different reverse primer for B. aphidicola (Buch_R_CV2:

186 CCCCCACTTTRGTTTTTCAAC) which resulted in a shorter product enabling us to 187 multiplex the PCRs. We confirmed that results were consistent between both primer 188 pairs. For aphids positive for $H$. defensa we additionally amplified part of the murE gene 189 (forward primer: murE16F: ACTAACGGGAAAACCACTAATAC \& reverse primer: 
murE936R: TTGAGAATGTCAGCGGTAATC) (Henry et al. 2013) and sent the amplicons to Microsynth (Balgach, Switzerland) for sequencing. The murE sequences differ between the three strains we used (Cayetano et al. 2015), enabling us to determine the strain present in each $H$. defensa positive aphid.

\section{Statistical Analysis}

All analyses and plot generation took place in R, version 3.6.0 (R Core Team 2019). To investigate treatment effects on population dynamics, we analyzed the number of aphids and the number of mummies and mummification rate as a function of the factors treatment, generation and their interaction, using linear mixed models (LMM) from the Ime4 package (Bates et al. 2015). Cage (replicate) was included as a random effect to account for the non-independence of successive counts from the same replicate populations. We also analyzed plant size with a LMM, as it may be affected by aphid population size. If the treatment $\times$ generation interaction was not significant $(p>0.05)$, it was removed from the models. The variance among treatments was further partitioned into the difference between cages with and without parasitoids, with three or one wasp genotype, and the variation among all parasitoid treatments, using linear orthogonal contrasts. To conform with the assumption of normality, aphid and mummy numbers and mummification rate were transformed using the transformTukey function from the rcompanion package (Mangiafico 2019) with a lambda of 0.75 for aphids, 0.375 for mummy number, and 0.325 for mummification rate.

$$
\text { To investigate changes of the aphid population composition in response to our }
$$

treatments, we analyzed the proportions of $H$. defensa-infected aphids and of aphid lines with each H. defensa haplotype (H15, H76, H402) with similar LMMs. To conform to the assumption of normality proportions were transformed by using the arc-sine of its 
214 square root except for the proportion of H15 where we used a power of 0.1 prior to

215 calculating arc-sine. Because $H$. defensa became fixed in some treatments at the end of

216 the experiment, we added one individual from each line to one replicate of every

217 generation in the dataset prior to analysis to avoid problems with model fitting. If

218 anything, this should have reduced differences and hence made comparisons more

219 conservative. Each model was followed with a type III analysis of variance (anova) using

220 ImerTest to obtain p-values for fixed effects with Satterthwaite's degrees of freedom

221 method (Kuznetsova et al. 2017). Significant differences between treatments with wasps

222 were followed up with post hoc tests using emmeans (Lenth 2019).

223 Finally, to analyze changes in the diversity of aphid lines (i.e. H. defensa absence or

224 haplotype), we calculated the Shannon index for each replicate (including the additional

225 individual in one replicate from each line, see above) using the R package vegan

226 (Oksanen et al. 2019). The Shannon index was then used as response in a linear mixed

227 model similar to the ones described above. We also calculated beta diversity across

228 replicates of the same treatment with divEst by estimating diversity and partitioning it

229 into alpha and beta diversity (entropart package; Marcon \& Hérault 2015). divEst

230 calculates bootstrap confidence intervals by drawing simulated communities from a

231 multinomial distribution that follows the observed frequencies which allowed us to

232 calculate lower and upper 95\% confidence intervals.

$233 \quad$ Results

234 Parasitoid wasps selected strongly against $H$. defensa-free aphids, such that they

235 virtually disappeared over time in all treatments containing wasps $(\mathrm{p}<0.0001$, Fig. $2 \mathrm{~A}$,

236 Tables 1A, S2). In the treatment without wasps, on the other hand, the H. defensa-free 
aphids increased over time and reached over $80 \%$ by the end of the experiment $(\mathrm{p}<0.001$, Tables S3-S4). The presence of wasps diminished aphid numbers $(\mathrm{p}<0.0001$, Fig. 3A, Tables 1F, S6), but this did not entail significant variation in plant size among treatments ( $p=0.1608$, Fig. 3B, Tables 1G, S6).

Concurrent with the increase of $H$. defensa-protected aphids in all cages with

242 parasitoids, the number of mummies and mummification rate began to decline after the

243 first two to three generations of selection ( $p<0.001$, Fig. 3C-D, Tables $1 \mathrm{H}-\mathrm{I}, \mathrm{S} 6)$. However,

244 the trajectories of these declines differed among treatments $(\mathrm{p}<0.001$, Tables $1 \mathrm{H}-\mathrm{I}, \mathrm{S} 6)$.

245 In two treatments with single wasp genotypes (W64 and W369), the number of

246 mummies declined rapidly $(\mathrm{p}<0.04)$ and the mummification rate remained close to zero 247 after about four generations (Fig. 3C-D, Tables S7-S8), which is indicative of highly 248 resistant aphid populations. Accordingly, aphids grew to larger population sizes in 249 treatments with either of these wasp genotypes, especially W369, than in the other 250 treatments with wasps ( $\mathrm{p}<0.02$, Fig. 3A, Tables S8-S9). These two wasps lines indeed 251 selected strongly for a single (but different) H. defensa strain (W64 for H76, W369 for 252 H402), which became fixed in all replicates of the respective treatment (Fig. 2B-C, S1, 253 Tables 1B-C, S3-S5). The third treatment with a single wasp line (W348) was somewhat 254 different in that two H. defensa strains (H76 \& H402) persisted until the end of the 255 experiment (Fig. 2B-C, Tables S3-S5), although this was mainly due to a single replicate 256 that became fixed for aphids with $\mathrm{H} 402$, whereas the other replicates became 257 dominated by aphids carrying H76 (Fig. 4). The two H. defensa strains H76 and H402 258 were also maintained in both treatments applying all wasp lines, either simultaneously 259 or sequentially (Fig. 2B-C, Tables S3-S5). Again, several replicates became fixed for one 260 or the other strain (Fig. 4), but both strains persisted in both treatments. Accordingly, 
261 H76 and H402 were significantly more frequent in the presence of the wasp genotype

262 against which they conferred strong resistance (W64 and W369) than in any of the

263 other treatments ( $\mathrm{p}<0.05$, Fig. 2B-C, S1, Tables S4-S5). Strain H15 was eliminated from

264 all treatments with parasitoids, but it remained present across generations in the

265 parasitoid-free treatment and hence showed a non-significant trend to be more frequent

266 without wasps ( $\mathrm{p}=0.0837$, Fig. 2D, Tables 1D, S2).

267 The unequal selection exerted by the different wasp treatments was also reflected in

268 aphid population diversity. Because all populations started at maximal diversity (4 lines

269 at equal proportions), Shannon diversity declined in all treatments $(p<0.0001$, Fig. $2 E$,

270 Tables 1E, S2). While diversity dropped to zero in two out of three treatments with

271 single wasp genotypes, it did not drop so low in the two mixed wasp treatments, and it

272 remained highest in the parasitoid-free treatment, where no aphid line was eliminated

273 completely (Fig. 2E, S1). However the main effect of treatment on Shannon diversity was

274 not statistically significant ( $p=0.0811$, Tables 1E, S2). Beta diversity, on the other hand,

275 increased from the initial minimum for all treatments, but it returned to a minimal level

276 in the treatments where a single wasp line consistently selected for one $H$. defensa strain

277 (W64 \& W369), while it stayed relatively high for the two mixed treatments as well as

278 treatment W348 and the wasp-free treatment (Fig. 2F, S1, Tables 2, S10). In those wasp

279 treatments that maintained $H$. defensa strain diversity across generations, the number of 280 mummies and mummification rate by parasitoids remained higher than in the

281 treatments that resulted in fixation of a single $H$. defensa strain (Fig. 3C-D, Tables S3-S4). 
Parasitoid wasp diversity strongly influenced endosymbiont diversity in our

284 laboratory host populations. In the presence of wasps, $H$. defensa increased rapidly in

285 frequency and approached fixation within just a few generations. Two wasp genotypes

286 selected strongly for single (but different) strains of $H$. defensa, such that a higher strain

287 diversity of symbionts was maintained when these wasps occurred in mixtures of wasp

288 genotypes rather than alone.

289 Without wasps, $H$. defensa frequencies decreased in the aphid populations, with strain

290 H15 possibly persisting somewhat longer than the other two strains (Fig. 4). It seems

291 likely that given more time, $H$. defensa would have died out completely. This reflects the

292 known fitness costs $H$. defens $a$ induces by reducing reproduction and longevity of aphid

293 hosts (Vorburger \& Gouskov 2011; Cayetano et al. 2015; Martinez et al. 2018; Sochard et

294 al. 2019). Costs to the host vary among different strains of $H$. defensa, which may at least

295 partly be related to different infection intensities (titers) achieved by different strains.

296 Our experiment confirms that these costs are strong enough to drive $H$. defensa (nearly)

297 to extinction when they are not offset by any benefits.

298 The benefit offsetting the costs in our experiment was the strong resistance $H$. defensa

299 provides against parasitoids, in agreement with previous studies on defensive

300 symbionts in captive insect populations (Herzog et al. 2007; Oliver et al. 2008; Xie et al.

3012015 ; Käch et al. 2018). The selection for symbiont-conferred protection led to increased

302 population-level resistance, as shown by the corresponding decrease in mummification,

303 albeit this decrease and hence the protective effect of $H$. defensa differed greatly

304 between treatments (i.e. wasps). Wasp identity, composition, and diversity were crucial 305 in deciding which $H$. defensa strains persisted. In two of the three single wasp line 
treatments, namely W369 and W64, a single, but different $H$. defensa strain quickly went

to fixation and any symbiont diversity was lost. The remaining strain of $H$. defensa

308 provided effective protection as illustrated by the parallel drop in mummification and

309 the limited impact parasitoids had on aphid numbers (Fig. 3). This confirms the

310 previously observed specificity of $H$. defensa protection (Rouchet \& Vorburger 2012;

311 Schmid et al. 2012; Cayetano \& Vorburger 2013, 2015; Cayetano et al. 2015; McLean \&

312 Godfray 2015; Leclair et al. 2016; Vorburger \& Rouchet 2016) and shows that without

313 any wasp diversity, $H$. defensa diversity can be lost rapidly. The remaining aphids were

314 so well protected against the specific wasp strain that without us resupplying

315 individuals every generation, the wasps would have been unlikely to persist (see Käch et

316 al. 2018). There is good evidence that, given enough time, parasitoid populations can

317 adapt to symbiont-conferred resistance (Dion et al. 2011; Dennis et al. 2017), but by

318 using asexual lines and supplying parasitoids every generation anew, we precluded this

319 possibility in the present experiment. In nature, of course, neither aphids nor

320 parasitoids occur in closed populations. Spatial or temporal refuges can shelter $H$.

321 defensa-free aphids from parasitism, local extinctions of parasitoids may allow their re-

322 immigration and population growth, which in turn would favour the re-establishment of

323 wasps. The interplay of these processes may well result in the intermediate $H$. defensa

324 frequencies observed in nature (Russell et al. 2013; Smith et al. 2015; Vorburger \&

325 Rouchet 2016; Zytynska \& Weisser 2016).

326 The third single wasp line treatment, W348, also selected strongly for $H$. defensa, but

327 it did not result in the complete fixation of a single strain of $H$. defensa. This line of $L$.

328 fabarum seems to have the ability to parasitize aphids harbouring all $H$. defensa strains

329 included in this study (Vorburger \& Rouchet 2016), although it exerted the strongest 
selection for H76 (Fig. 2). Only one replicate became dominated by H402 (Fig. 4).

331 Considering the limited number of founders and individuals transferred every

332 generation, some degree of stochasticity in the outcome is expected when no strain is

333 fully protective or clearly inferior in protecting hosts, such as H15, which disappeared

334 from all cages containing wasps. Given more time, H76 may have become fixed at least in

335 those replicates where it did not disappear initially. Nevertheless, we cannot rule out

336 that this parasitoid's ability to overcome resistance by different symbiont strains

337 resulted in less biased selection enabling the maintenance of symbiont diversity for a

338 longer period, similar to a mixture of wasps (see below).

339 Both of the H. defensa strains $\mathrm{H76}$ and $\mathrm{H} 402$ were also maintained when the

340 parasitoids comprised a mixture of the parasitoid lines, either simultaneously or

341 sequentially. Each strain provided effective protection against a different wasp line;

342 their effects on host fitness were therefore more balanced in the presence of multiple

343 lines of wasps. However, this was not evident within every cage. Many replicates became

344 dominated by one of the strains (Fig. 4), likely reflecting the role of random drift, but

345 across replicates two $H$. defensa strains persisted, such that beta diversity remained high

346 in both mixed parasitoid treatments (Fig. 2E). Our findings thus show that parasitoid

347 diversity can indeed promote symbiont diversity in host populations. In a field study

348 manipulating plant diversity, Zytynska et al. (2016) observed that higher plant diversity

349 resulted in higher symbiont diversity in aphid populations colonizing these plants. The

350 authors speculated that this could, among other factors, be mediated by parasitoid

351 diversity, because parasitoid diversity tends to increase with higher plant diversity (e.g.

352 Petermann et al. 2010). The results of our laboratory study would indeed be consistent

353 with this conjecture. 
We focused exclusively on aphids infected with a single facultative endosymbiont, but

355 in nature individual aphids can harbour multiple symbionts (McLean et al. 2016; Zytynska \& Weisser 2016; Guo et al. 2017). Multiple infections are relatively rare in the black bean aphid (Vorburger \& Rouchet 2016), but they can be very common in other aphid species such as the pea aphid, Acyrthosiphon pisum. Coinfections may alter costs and benefit in ways not necessarily predictable from single infections (McLean et al. 2018; Weldon et al. 2019), but generally the defense provided by protective symbionts is retained, albeit often at higher costs to the hosts (Doremus \& Oliver 2017; Leclair et al. 2017; McLean et al. 2018).

Biologists have long used genetic interaction models to study antagonistic coevolution and the resulting maintenance of diversity. Classical examples include the gene for gene model, initially based on plant-pathogen interactions, where each resistant allele in the host has to be countered by a virulent allele in the corresponding locus of the parasite for successful infection (Flor 1956) and the matching alleles model, initially inspired by the vertebrate immune system, where the parasites require the alleles that exactly match the appropriate resistance alleles in a host (Frank 1993). Further possible models exist on a continuum between these endpoints (Agrawal \& Lively 2002), and the same framework can be applied to protective symbionts (Kwiatkowski et al. 2012). The patterns of high specificity we observed fit well with the matching alleles model, albeit some parasitoids (e.g. W348) seem more generalised in overcoming resistance. Under a matching allele model, variation in frequencies of different parasites will result in an increase in symbionts providing resistance against the most frequent parasite type, which in turn selects for other parasites able to overcome this resistance. Cycling between different parasite genotypes (or species) and 
different protective symbionts will ensue as long as the population size is large enough or re-immigration prevents complete extinction. Such Red Queen dynamics are fundamental to the co-evolution of antagonists and help maintain variation in host defences, such as immune genes, as well as parasite offenses (reviewed e.g. by Ebert 2008; Brockhurst \& Koskella 2013; Brockhurst et al. 2014; Strotz et al. 2018). Here we could show that, akin to the role parasites play in shaping an animal's own immune system, they can also play a crucial role in maintaining the diversity of protective symbionts. An important difference remains, though. Immune genes are reshuffled and inherited biparentally, such that animals have the potential to shape their offspring's immune system through adaptive mate choice. By contrast, symbionts are usually transmitted maternally without mixing, albeit routes of horizontal transmission for heritable symbionts exist, such as being vectored by parasitoids (Gehrer \& Vorburger 2012). Nevertheless, such events are likely to be rare and it is currently unknown whether they play a significant role in this system (Hafer \& Vorburger 2019). Our observations have applied implications as well, because parasitoids are frequently used to control pest aphids in greenhouse crops. The effectiveness of parasitoid release may be strongly reduced in the presence of $H$. defensa (Käch et al. 2018), but using multiple or alternating wasp lines and resupplying them regularly may maintain parasitoid diversity and hence increase the chances of successful biocontrol if pests harbour multiple protective symbionts. In medicine, similar strategies are used to overcome the evolution of antibiotic resistance, for example by using multiple antibiotics simultaneously or sequentially (e.g. Bonhoeffer et al. 1997; Sun et al. 2010; Baym et al. 2016). 
401 Protective symbionts are widespread throughout the animal kingdom and not only

402 protect against parasites. Some symbionts can also protect against predators, for

403 example (reviewed by Flórez et al., 2015). Do antagonistic interactions with a variety of

404 natural enemies also promote the diversity of symbionts in other systems? The answer

405 to this question will depend on how targeted microbial defenses are. The specificity seen

406 in the H. defensa-L. fabarum interaction is certainly not unique, even though its degree

407 may be unusual. Protection by microbial symbionts frequently relies on the production

408 of chemicals with narrow toxicity to certain predators and parasites, or with specific

409 antifungal, antibacterial, or antiviral properties (Flórez et al. 2015). That the diversity of

410 an organism's microbial protectors is shaped by the diversity of its natural enemies may

411 therefore be a very general phenomenon.

412

413 
We would like to thank Paula Rodriguez, Silvan Rossbacher, Raffael Stegmayer, and 8471/1-1), and CV acknowledges support by the Swiss National Science Foundation 418 (grants nr. CRSII3_154396 and 21003A_181969).

\section{References}

Agrawal, A. \& Lively, C.M. (2002). Infection genetics: Gene-for-gene versus matching-

421 alleles models and all points in between. Evol. Ecol. Res., 4, 79-90.

Apanius, V., Penn, D., Slev, P.R., Ruff, L.R. \& Potts, W.K. (1997). The Nature of Selection on the Major Histocompatibility Complex. Crit. Rev. Immunol., 17, 179-224. (2014). Specialisation of bacterial endosymbionts that protect aphids from parasitoids. Ecol. Entomol., 39, 736-739.

Bates, D.M., Maechler, M., Bolker, B.M. \& Walker, S. (2015). Fitting linear mixed-effects models using lme4. J. Stat. Softw., 67, 1-48.

Baym, M., Stone, L.K. \& Kishony, R. (2016). Multidrug evolutionary strategies to $430 \quad$ reverse antibiotic resistance. Science., 351, aad3292.

431 Bernatchez, L. \& Landry, C. (2003). MHC studies in nonmodel vertebrates: What have 432 we learned about natural selection in 15 years? J. Evol. Biol., 16, 363-377.

433 Bhaya, D., Davison, M. \& Barrangou, R. (2011). CRISPR-Cas Systems in Bacteria and 434 Archaea: Versatile Small RNAs for Adaptive Defense and Regulation. Annu. Rev. Genet., 435 45, 273-297. 
Bonhoeffer, S., Lipsitch, M. \& Levin, B.R. (1997). Evaluating treatment protocols to prevent antibiotic resistance. Proc. Natl. Acad. Sci. U. S. A., 94, 12106-12111. (2014). Running with the Red Queen: The role of biotic conflicts in evolution. Proc. $R$. $440 \quad$ Soc. B Biol. Sci., 281, 20141382.

441 Brockhurst, M.A. \& Koskella, B. (2013). Experimental coevolution of species 442 interactions. Trends Ecol. Evol., 28, 367-375.

443 Brownlie, J.C. \& Johnson, K.N. (2009). Symbiont-mediated protection in insect hosts. 444 Trends Microbiol., 17, 348-354.

445 Cayetano, L., Rothacher, L., Simon, J. \& Vorburger, C. (2015). Cheaper is not always 446 worse: strongly protective isolates of a defensive symbiont are less costly to the aphid 447 host. Proc. R. Soc. B, 282, 20142333.

448 Cayetano, L. \& Vorburger, C. (2013). Genotype-by-genotype specificity remains robust 449 to average temperature variation in an aphid/endosymbiont/parasitoid system. J. Evol. 450 Biol., 26, 1603-1610.

451 Cayetano, L. \& Vorburger, C. (2015). Symbiont-conferred protection against 452 Hymenopteran parasitoids in aphids: How general is it? Ecol. Entomol., 40, 85-93.

453 Dennis, A.B., Patel, V., Oliver, K.M. \& Vorburger, C. (2017). Parasitoid gene expression 454 changes after adaptation to symbiont-protected hosts. Evolution., 71, 2599-2617.

455 Dion, E., Zélé, F., Simon, J.C. \& Outreman, Y. (2011). Rapid evolution of parasitoids 456 when faced with the symbiont-mediated resistance of their hosts. J. Evol. Biol., 24, 741$457 \quad 750$.

458 Doremus, M.R. \& Oliver, K.M. (2017). Aphid heritable symbiont exploits defensive 459 mutualism. Appl. Environ. Microbiol., 83, e03276-16. 
Ebert, D. (2008). Host-parasite coevolution: Insights from the Daphnia-parasite model system. Curr. Opin. Microbiol., 11, 290-301.

Ferrari, J., West, J. a, Via, S., Godfray, H.C.J. \& Al, E.T. (2012). Population genetic structure ans secondary symbionts in host-associated populations of the pea aphid. Evolution., 66, 375-390.

Flor, H.H. (1956). The Complementary Genic Systems in Flax and Flax Rust. Adv. Genet., 8, 29-54.

Flórez, L. V, Biedermann, P.H.W., Engl, T. \& Kaltenpoth, M. (2015). Defensive symbioses of animals with prokaryotic and eukaryotic microorganisms. Nat. Prod. Rep., $32,904-936$.

Frank, S.A. (1993). Specificity versus detectable polymorphism in host-parasite genetics. Proc. R. Soc. B Biol. Sci., 254, 191-197.

Fukatsu, T. \& Nikoh, N. (1998). Two intracellular symbiotic bacteria from the mulberry psyllid Anomoneura mori (insecta Homoptera). Appl. Environ. Microbiol., 64, 3599-3606.
Garrett, R.A., Vestergaard, G. \& Shah, S.A. (2011). Archaeal CRISPR-based immune systems: Exchangeable functional modules. Trends Microbiol., 19, 549-556.

$$
\text { Ghosh, J., Lun, C.M., Majeske, A.J., Sacchi, S., Schrankel, C.S. \& Smith, L.C. (2011). }
$$

Invertebrate immune diversity. Dev. Comp. Immunol., 35, 959-974.

Gouskov, A., Reyes, M., Wirthner-Bitterlin, L. \& Vorburger, C. (2016). Fish population genetic structure shaped by hydroelectric power plants in the upper Rhine catchment. Evol. Appl., 9, 394-408.

Guo, J., Hatt, S., He, K., Chen, J., Francis, F. \& Wang, Z. (2017). Nine facultative endosymbionts in aphids. A review. J. Asia. Pac. Entomol., 20, 794-801. 
Hafer, N. \& Vorburger, C. (2019). Diversity begets diversity: do parasites promote

485

486

487

488

489

490

491

492

493

494

495

496

497

498

499

500

501

502

503

504

505

506

507

variation in protective symbionts? Curr. Opin. Insect Sci., 32, 8-14.

Henry, L.M., Peccoud, J., Simon, J.C., Hadfield, J.D., Maiden, M.J.C., Ferrari, J., et al. (2013). Horizontally transmitted symbionts and host colonization of ecological niches. Curr. Biol., 23, 1713-1717.

Herzog, J., Muller, C.B. \& Vorburger, C. (2007). Strong parasitoid-mediated selection in experimental populations of aphids. Biol. Lett., 3, 667-669.

Heyworth, E.R. \& Ferrari, J. (2015). A facultative endosymbiont in aphids can provide diverse ecological benefits. J. Evol. Biol., 28, 1753-1760.

Horvath, P., Barrangou, R., Horvath1, P. \& Barrangou2, R. (2010). CRISPR/Cas, the Immune System of Bacteria and Archaea. Science., 327, 167-170.

Käch, H., Mathé-Hubert, H., Dennis, A.B. \& Vorburger, C. (2018). Rapid evolution of symbiont-mediated resistance compromises biological control of aphids by parasitoids. Evol. Appl., 11, 220-230.

Kuznetsova, A., Brockhoff, P.B. \& Christensen, R.H.B. (2017). lmerTest Package: Tests in Linear Mixed Effects Models. J. Stat. Softw., 82, 1-26.

Kwiatkowski, M., Engelstädter, J. \& Vorburger, C. (2012). On Genetic Specificity in Symbiont-Mediated Host-Parasite Coevolution. PLoS Comput. Biol., 8, e1002633.

Leclair, M., Polin, S., Jousseaume, T., Simon, J.C., Sugio, A., Morlière, S., et al. (2017). Consequences of coinfection with protective symbionts on the host phenotype and symbiont titres in the pea aphid system. Insect Sci., 24, 798-808.

$$
\text { Leclair, M., Pons, I., Mahéo, F., Simon, J.-C. \& Outreman, Y. (2016). Diversity in }
$$
symbiont consortia in the pea aphid complex is associated with large phenotypic variation in the insect host. Evol. Ecol., 30, 925-941. 
Lenth, R. (2019). emmeans: Estimated Marginal Means, aka Least-Squares Means. https://cran.r-project.org/package=emmeans. mechanisms of immune receptor diversity. Curr. Opin. Immunol., 19, 526-534.

513 Program Evaluation. https://cran.r-project.org/package=rcompanion.

514 Marcon, E. \& Hérault, B. (2015). Entropart: An R package to measure and partition 515 diversity. J. Stat. Softw., 67, 1-26.

516 Martinez, A.J., Doremus, M.R., Kraft, L.J., Kim, K.L. \& Oliver, K.M. (2018). Multi-modal 517 defences in aphids offer redundant protection and increased costs likely impeding a 518 protective mutualism. J. Anim. Ecol., 87, 464-477.

519 McLean, A.H.C. \& Godfray, H.C.J. (2015). Evidence for specificity in symbiont520 conferred protection against parasitoids. Proc. R. Soc. B, 282, 20150977.

521 McLean, A.H.C., Parker, B.J., Hrček, J., Henry, L.M. \& Godfray, H.C.J. (2016). Insect 522 symbionts in food webs. Philos. Trans. R. Soc. B, 371, 20150325.

523 McLean, A.H.C., Parker, B.J., Hrček, J., Kavanagh, J.C., Wellham, P.A.D. \& Godfray, H.C.J. 524 (2018). Consequences of symbiont co-infections for insect host phenotypes. J. Anim. 525 Ecol., 87, 478-488.

526 Messier-Solek, C., Buckley, K.M. \& Rast, J.P. (2010). Highly diversified innate receptor 527 systems and new forms of animal immunity. Semin. Immunol., 22, 39-47.

528 Milinski, M. (2006). The Major Histocompatibility Complex, Sexual Selection, and 529 Mate Choice. Annu. Rev. Ecol. Evol. Syst., 37, 159-186.

530 Moran, N.A., Russell, J.A., Koga, R. \& Fukatsu, T. (2005). Evolutionary relationships of 531 three new species of Enterobacteriaceae living as symbionts of aphids and other insects. 
Appl. Environ. Microbiol., 71, 3302-3310.

Oksanen, J., Blanchet, F.G., Friendly, M., Kindt, R., Legendre, P., McGlinn, D., et al. (2019). vegan: Community Ecology Package. https://cran.r-project.org/package=vegan.

$$
\text { Oliver, K.M., Campos, J., Moran, N.A. \& Hunter, M.S. (2008). Population dynamics of }
$$
defensive symbionts in aphids. Proc. R. Soc. B, 275, 293-299.

$$
\text { Oliver, K.M., Degnan, P.H., Burke, G.R. \& Moran, N.A. (2010). Facultative Symbionts in }
$$
Aphids and the Horizontal Transfer of Ecologically Important Traits. Annu. Rev. Entomol., $55,247-266$.

$$
\text { Oliver, K.M., Russell, J.A., Moran, N.A. \& Hunter, M.S. (2003). Facultative bacterial }
$$
symbionts in aphids confer resistance to parasitic wasps. Proc. Natl. Acad. Sci., 100, 1803-1807.

$$
\text { Oliver, K.M., Smith, A.H. \& Russell, J.A. (2014). Defensive symbiosis in the real world - }
$$
advancing ecological studies of heritable, protective bacteria in aphids and beyond. Funct. Ecol., 28, 341-355.

$$
\text { Parker, B.J., Hrček, J., McLean, A.H.C. \& Godfray, H.C.J. (2017). Genotype specificity }
$$
among hosts, pathogens, and beneficial microbes influences the strength of symbiontmediated protection. Evolution., 71, 1222-1231.

$$
\text { Petermann, J.S., Müller, C.B., Weigelt, A., Weisser, W.W. \& Schmid, B. (2010). Effect of }
$$
plant species loss on aphid-parasitoid communities. J. Anim. Ecol., 79, 709-720.

$$
\text { Piertney, S.B. \& Oliver, M.K. (2006). The evolutionary ecology of the major }
$$
histocompatibility complex. Heredity., 96, 7-21.

$$
\text { R Core Team (2019). R: A language and environment for statistical computing. } R
$$

Foundation for Statistical Computing, Vienna, Austria. https://www.r-project.org/.

$$
\text { Rouchet, R. \& Vorburger, C. (2012). Strong specificity in the interaction between }
$$


parasitoids and symbiont-protected hosts. J. Evol. Biol., 25, 2369-2375.

Russell, J.A., Weldon, S., Smith, A.H., Kim, K.L., Hu, Y., Łukasik, P., et al. (2013).

Uncovering symbiont-driven genetic diversity across North American pea aphids. Mol.

Ecol., 22, 2045-2059.

Satar, G., Karacaoğlu, M., Uygun, N. \& Satar, S. (2019). Some Demographic Parameters

561 of Lysiphlebus confusus, L. fabarum, and L. testaceipes (Hymenoptera: Braconidae:

562 Aphidiinae). J. Econ. Entomol., 112, 1105-1111.

563 Schmid, M., Sieber, R., Zimmermann, Y.S. \& Vorburger, C. (2012). Development, 564 specificity and sublethal effects of symbiont-conferred resistance to parasitoids in 565 aphids. Funct. Ecol., 26, 207-215.

566 Smith, A.H., Łukasik, P., O’Connor, M.P., Lee, A., Mayo, G., Drott, M.T., et al. (2015).

567 Patterns, causes and consequences of defensive microbiome dynamics across multiple 568 scales. Mol. Ecol., 24, 1135-1149.

569 Sochard, C., Leclair, M., Simon, J.C. \& Outreman, Y. (2019). Host plant effects on the 570 outcomes of defensive symbioses in the pea aphid complex. Evol. Ecol., 33, 651-669.

571 Sommer, S. (2005). The importance of immune gene variability (MHC) in evolutionary 572 ecology and conservation. Front. Zool., 2, 16.

573 Sorek, R., Lawrence, C.M. \& Wiedenheft, B. (2013). CRISPR-Mediated Adaptive 574 Immune Systems in Bacteria and Archaea. Annu. Rev. Biochem. 2013. 82, 82, 237-266. 575 Spurgin, L.G. \& Richardson, D.S. (2010). How pathogens drive genetic diversity: MHC, 576 mechanisms and misunderstandings. Proc. R. Soc. B, 277, 979-988.

577 Strotz, L.C., Simões, M., Girard, M.G., Breitkreuz, L., Kimmig, J. \& Lieberman, B.S. 578 (2018). Getting somewhere with the red queen: Chasing a biologically modern definition 579 of the hypothesis. Biol. Lett., 14, 20170734. 
583 Cytochrome I-II in Aphids of the Genus Sitobion (Hemiptera: Aphididae) Oxidase. Mol. 584 Biol., 13, 510-524.

585 Tomanović, Ž., Mitrović, M., Petrović, A., Kavallieratos, N.G., Žikić, V., Ivanović, A., et al. 586 (2018). Revision of the European Lysiphlebus species (Hymenoptera: Braconidae:

587 Aphidiinae) on the basis of COI and 28SD2 molecular markers and morphology. 588 Arthropod Syst. Phylogeny, 76, 179-213.

589 Vorburger, C. (2014). The evolutionary ecology of symbiont-conferred resistance to 590 parasitoids in aphids. Insect Sci., 21, 251-264.

591 Vorburger, C. \& Gouskov, A. (2011). Only helpful when required: A longevity cost of 592 harbouring defensive symbionts. J. Evol. Biol., 24, 1611-1617.

593 Vorburger, C. \& Perlman, S.J. (2018). The role of defensive symbionts in host-parasite 594 coevolution. Biol. Rev., 93, 1747-1764.

595 Vorburger, C. \& Rouchet, R. (2016). Are aphid parasitoids locally adapted to the 596 prevalence of defensive symbionts in their hosts? BMC Evol. Biol., 16, 271.

597 Watson, F.L., Watson, F.L., Pu, R., Kondo, M., Rebel, V.I. \& Schmucker, D. (2005).

598 Extensive Diversity of Ig-Superfamily Proteins in the Immune System of Insects. Science., $599 \quad 309,1874-1878$.

600 Wegner, K.M., Kalbe, M., Schaschl, H. \& Reusch, T.B.H. (2004). Parasites and individual 601 major histocompatibility complex diversity - An optimal choice? Microbes Infect., 6, $602 \quad 1110-1116$.

603 Weldon, S.R., Russell, J.A. \& Oliver, K.M. (2019). More is not always better: 
604 coinfections with defensive symbionts generate highly variable outcomes. Appl. Environ. 605 Microbiol., 86, e02537-19.

606 Xie, J., Winter, C., Winter, L. \& Mateos, M. (2015). Rapid spread of the defensive 607 endosymbiont Spiroplasma in Drosophila hydei under high parasitoid wasp pressure.

608 FEMS Microbiol. Ecol., 91, 1-11.

609 Zytynska, S.E., Meyer, S.T., Sturm, S., Ullmann, W., Mehrparvar, M. \& Weisser, W.W.

610 (2016). Secondary bacterial symbiont community in aphids responds to plant diversity.

611 Oecologia, 180, 735-747.

612 Zytynska, S.E. \& Weisser, W.W. (2016). The natural occurrence of secondary bacterial 613 symbionts in aphids. Ecol. Entomol., 41, 13-26.

614

615 
Table 1: Linear mixed effect models. Note that non-significant interactions have been removed from the

618 final models and in these cases, statistics for treatment and generation are for the model without

619 interaction. Significant p-values are highlighted in bold.

\begin{tabular}{|c|c|c|c|}
\hline factor & df & $\mathbf{F}$ & $\mathbf{p}$ \\
\hline \multicolumn{4}{|c|}{ A: Proportion of $H$. defensa } \\
\hline Treatment & 5,24 & 11.800 & $<0.0001$ \\
\hline Generation & 3,71 & 32.010 & $<0.0001$ \\
\hline Treatment: Generation & 15,71 & 8.220 & $<0.0001$ \\
\hline \multicolumn{4}{|c|}{ B: Proportion of H76 } \\
\hline Treatment & 5,23 & 8.040 & 0.0002 \\
\hline Generation & 3,71 & 3.080 & 0.0329 \\
\hline Treatment: Generation & 15,71 & 4.250 & $<0.0001$ \\
\hline \multicolumn{4}{|c|}{ C: Proportion of $\mathrm{H402}$} \\
\hline Treatment & 5,24 & 8.340 & 0.0001 \\
\hline Generation & 3,71 & 0.320 & 0.8131 \\
\hline Treatment: Generation & 15,71 & 3.780 & 0.0001 \\
\hline \multicolumn{4}{|c|}{ D: Proportion of H15 } \\
\hline Treatment & 5,24 & 2.230 & 0.0837 \\
\hline Generation & 3,86 & 33.840 & $<0.0001$ \\
\hline Treatment: Generation & 15,71 & 1.360 & 0.1931 \\
\hline \multicolumn{4}{|c|}{ E: Shannon diversity } \\
\hline Treatment & 5,24 & 2.270 & 0.0802 \\
\hline Generation & 3,86 & 146.850 & $<0.0001$ \\
\hline Treatment: Generation & 15,71 & 0.830 & 0.6418 \\
\hline \multicolumn{4}{|c|}{ F: Aphid number } \\
\hline Treatment & 5,24 & 19.420 & $<0.0001$ \\
\hline Generation & 9,258 & 17.380 & $<0.0001$ \\
\hline Treatment: Generation & 45,213 & 1.210 & 0.1887 \\
\hline \multicolumn{4}{|c|}{ G: Plant size } \\
\hline Treatment & 5,24 & 1.750 & 0.1608 \\
\hline Generation & 9,258 & 19.780 & $<0.0001$ \\
\hline Treatment: Generation & 45,214 & 0.610 & 0.9761 \\
\hline \multicolumn{4}{|c|}{ H: Mummy number } \\
\hline Treatment & 4,20 & 8.840 & 0.0003 \\
\hline Generation & 9,177 & 34.510 & $<0.0001$ \\
\hline Treatment: Generation & 36,177 & 2.550 & $<0.0001$ \\
\hline \multicolumn{4}{|c|}{ I: Mummification rate } \\
\hline Treatment & 4,19 & 28.950 & $<0.0001$ \\
\hline Generation & 9,173 & 18.940 & $<0.0001$ \\
\hline Treatment: Generation & 36,173 & 2.930 & $<0.0001$ \\
\hline
\end{tabular}


621 Table 2: Beta diversity for each treatment in the last (10th) generation. Estimated beta diversity and

622 Confidence intervals have been obtained through bootstrapping. For data from previous generations see

623 Table S10.

\begin{tabular}{|c|c|c|c|c|}
\hline treatment & beta, measured & beta, estimated & upper CI & lower CI \\
\hline NoWasp & 1.54 & 1.54 & 1.23 & 1.76 \\
\hline W348 & 2.14 & 2.14 & 2.13 & 2.49 \\
\hline W369 & 1 & 1 & 1 & 1 \\
\hline W64 & 1 & 1 & 1 & 1 \\
\hline WmixSeq & 1.60 & 1.62 & 1.49 & 1.83 \\
\hline WmixSim & 1.88 & 1.87 & 1.55 & 2.19 \\
\hline
\end{tabular}

624 


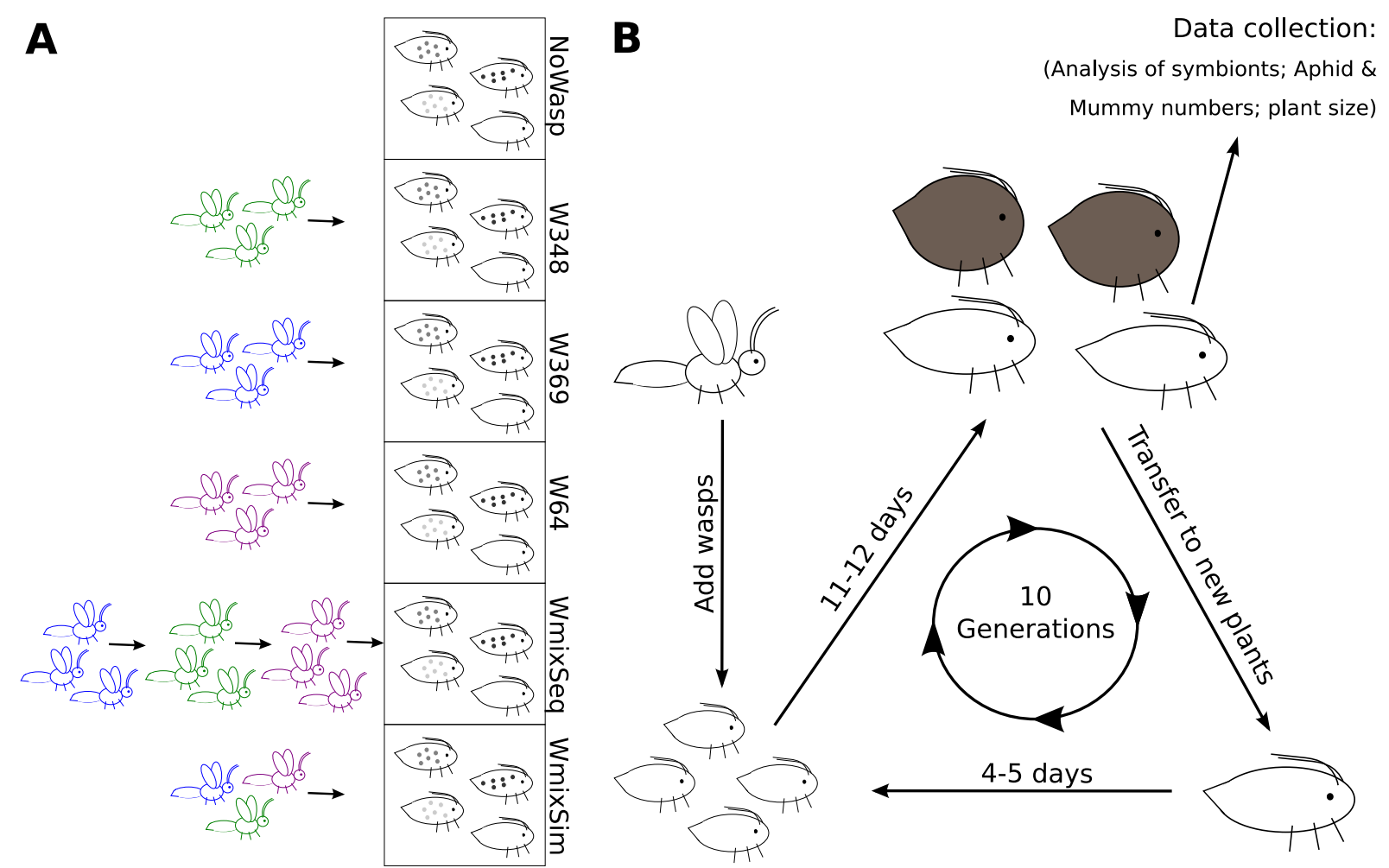

630 generation and analyze symbiont composition (after generation 2, 6, and 10 only), while mummified

631 aphids (brown) were discarded. The offspring of the transferred aphids was subsequently exposed to 632 parasitoid wasps to create selection for protective symbionts. 


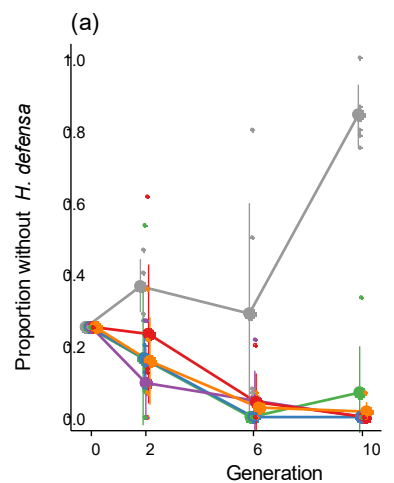

(e)

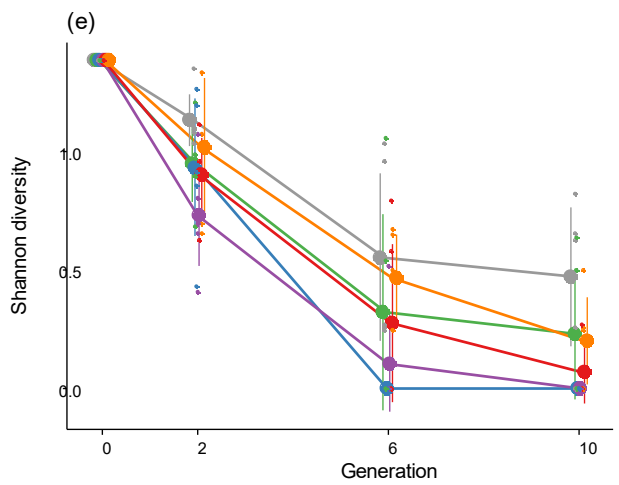

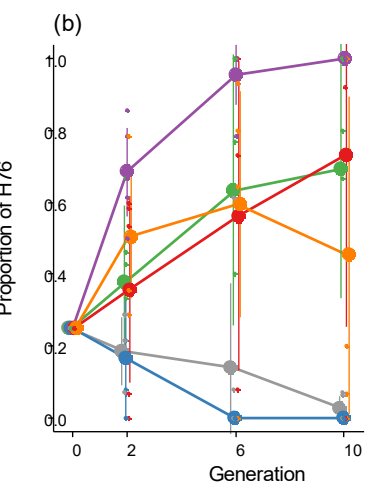
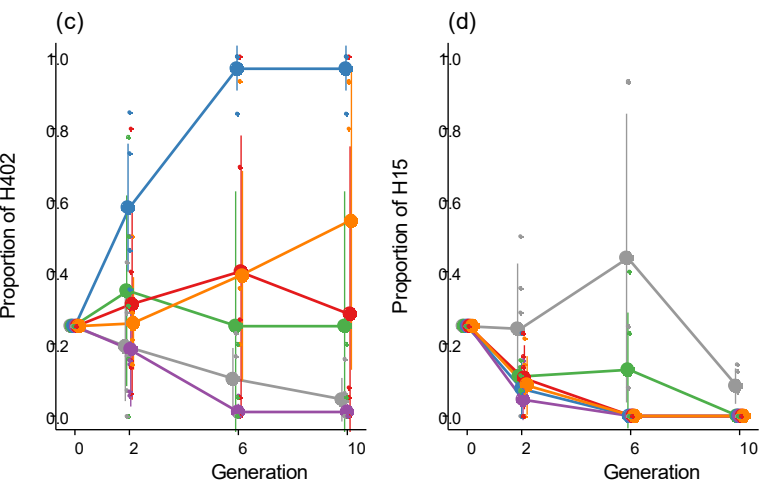

(f)
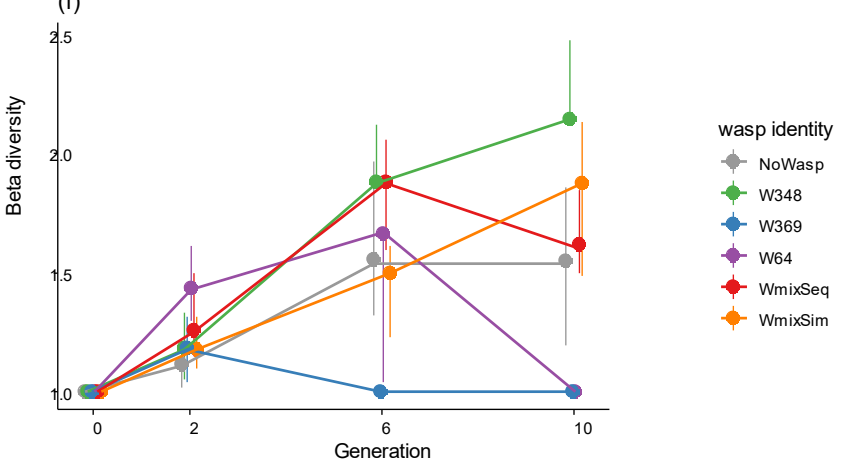

635

Figure 2: Changes in symbiont diversity and frequency of aphid lines with different $H$. defensa-

636 haplotypes for each treatment. A: H. defensa-free aphids, B: H. defensa-haplotype H76, C: H. defensa-

637 haplotype H402, D: H. defensa-haplotype H15, E: Shannon diversity, F: Beta diversity. Error bars indicate

$63895 \%$ CI. Confidence intervals for beta diversity have been obtained through bootstrapping. 
(a)
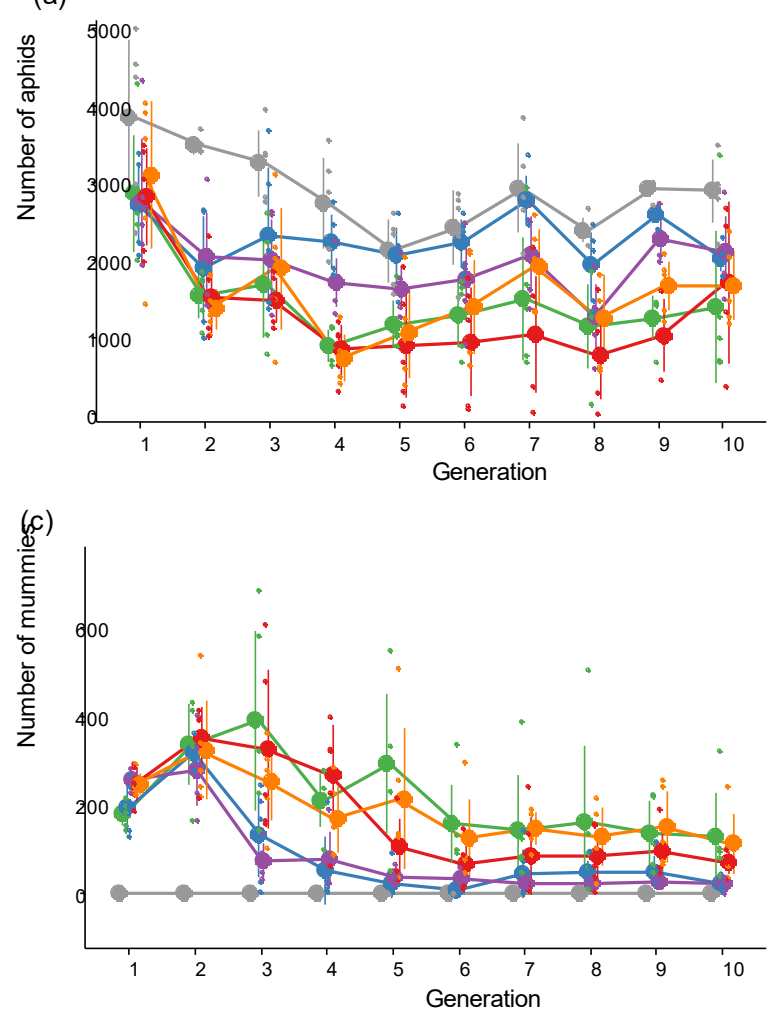

(b)

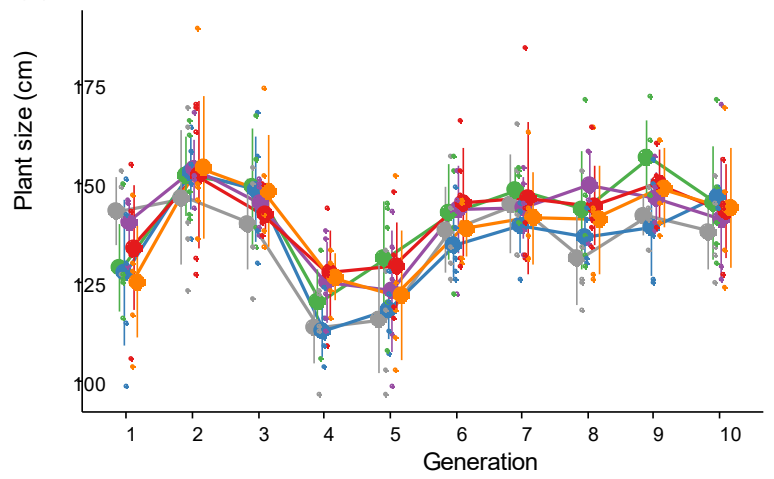

(d)

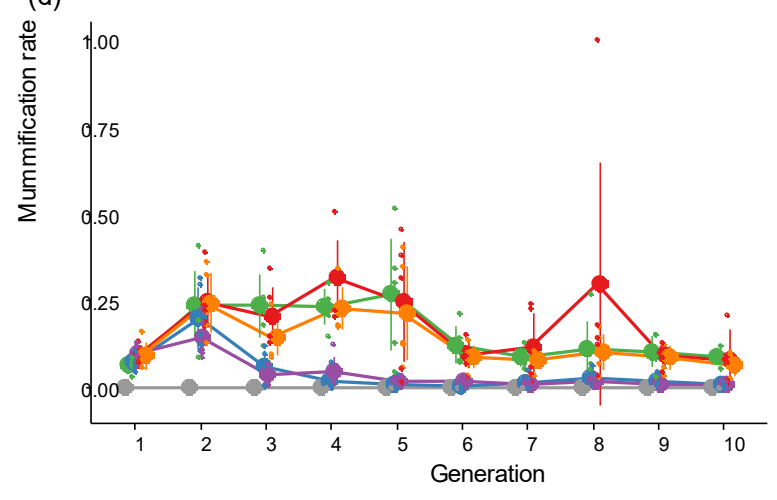

640

$$
\text { wasp identity } \begin{aligned}
& \phi \text { NoWasp }+ \text { w369 }+ \text { WmixSeq } \\
& \text { W348 }+ \text { W64 }+ \text { WmixSim }
\end{aligned}
$$

641 Figure 3: Aphid number (A), plant size (B), mummy number (C), and mummification rate (D). Error 642 bars indicate $95 \% \mathrm{CI}$.

643

644 


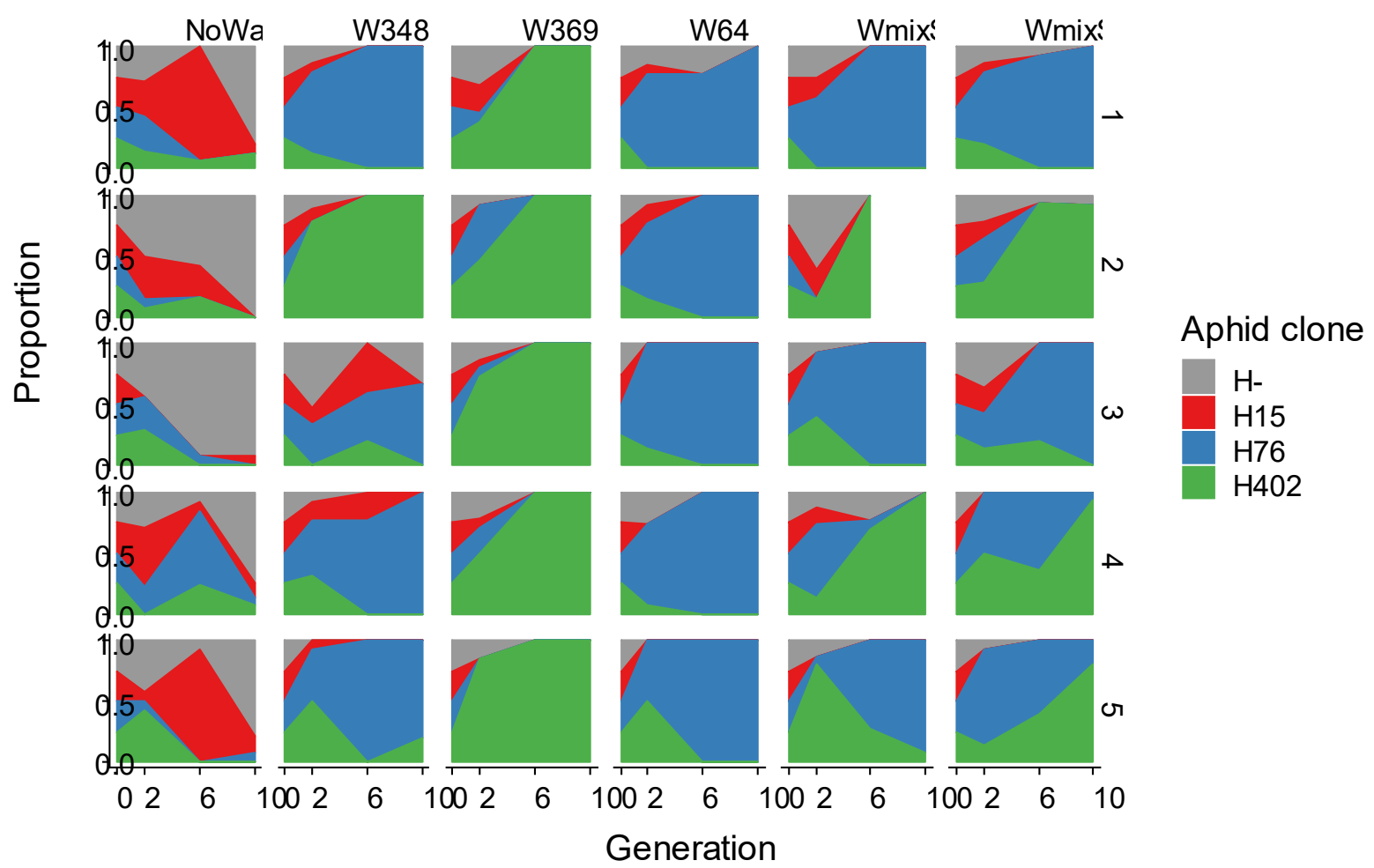

645

646 Figure 4: Changes in frequency of aphid clones with different $H$. defensa strains (or without $H$.

647 defensa) for each treatment (columns) and replicate (rows). Clone H-indicates H. defensa free-aphids,

648 clones H15, H76 and H402 aphids with different strains of $H$. defensa. 